\title{
Comparison of Coaxial Magnetic Gears With Different Topologies
}

\author{
Linni Jian, K. T. Chau, Yu Gong, J. Z. Jiang, Chuang Yu, and Wenlong Li \\ Department of Electrical and Electronic Engineering, The University of Hong Kong, Hong Kong, China
}

\begin{abstract}
This paper quantitatively compares two coaxial magnetic gears (CMGs) with different topologies, namely, the CMG installed with radially magnetized permanent magnets (CMGRM) and the CMG installed with Halbach magnetized permanent magnets (CMGHM). By using the 3D finite element method, the end-effect and the performances of both CMGs are investigated. Analysis results show that the CMGHM can offer higher pull-out torque, lower torque ripple and lower iron losses than the CMGRM. Experimental results are also given for verification.
\end{abstract}

Index Terms-Finite element method, Halbach arrays, magnetic gears, permanent magnet, torque transmission.

\section{INTRODUCTION}

$\mathbf{C}$ OAXIAL magnetic gears (CMGs) can offer some distinct advantages over mechanical gears, namely, minimum acoustic noise, free from maintenance, inherent overload protection, and physical isolation between input and output shafts [1]-[4]. They are particularly attractive for those applications desiring a high speed reduction ratio, such as electric vehicles [5] and wind generators [6]. The conventional CMG topology is installed with radially magnetized (RM) permanent magnets (PMs), hence termed the CMGRM. It is well known that Halbach magnetized (HM) PM arrays hold the attractive features of near-sinusoidal airgap flux density distribution, strong field intensity and good self-shielding magnetization [7]. Thus, the CMG with HM PM arrays, termed the CMGHM, has recently been proposed to further improve the performance [8]. However, the corresponding merits are only supported by analytical discussion and the $2 \mathrm{D}$ finite element method (FEM). Actually, since the CMGs are a pancake-like magnetic device, the end-effect should not be neglected when the torque transmission ability is evaluated.

The purpose of this paper is to quantitatively compare the two CMG topologies based on the 3D-FEM which takes the endeffect into account. The iron losses occurred in the iron yokes of the two rotors and the stationary ring will also be discussed. Most importantly, the corresponding prototypes will be tested and compared with the analysis results for verification.

\section{TORQUE TRANSMISSION}

Fig. 1 shows the topologies of the two CMGs. For both the CMGHM and CMGRM, the pole-pair number on the inner rotor $p_{1}$ is 4 , and that on the outer rotor $p_{2}$ equals 17 . The number of ferromagnetic segments on their stationary rings $N_{s}$ is equal to 21 , which is the sum of $p_{1}$ and $p_{2}$. The rotational speeds of the two rotors are governed by [1]:

$$
\omega_{2}=-\left(p_{1} / p_{2}\right) \omega_{1}
$$

Thus, the gear ratio of 17:4 is resulted. For the CMGHM, the arrays are designed with the numbers of PM segments per pole equal to 2 and 3 for the inner rotor and the outer rotor, respectively. Moreover, by artfully designing the magnetization direction of each PM segment in the CMGHM, the inner rotor

Manuscript received March 01, 2009. Current version published September 18, 2009. Corresponding author: L. Jian (e-mail: lnjian@eee.hku.hk).

Digital Object Identifier 10.1109/TMAG.2009.2021662

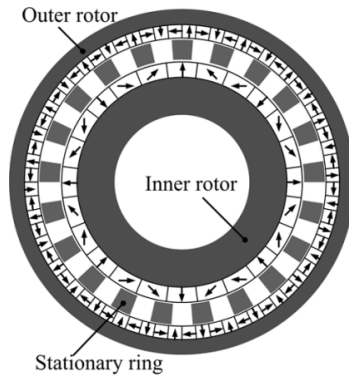

(a)

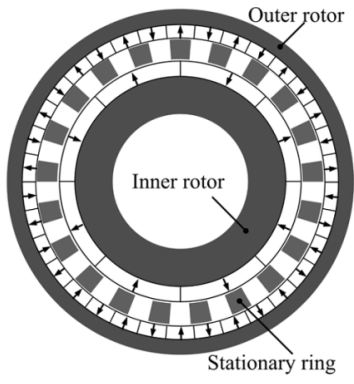

(b)
Fig. 1. Topologies. (a) CMGHM. (b) CMGRM.

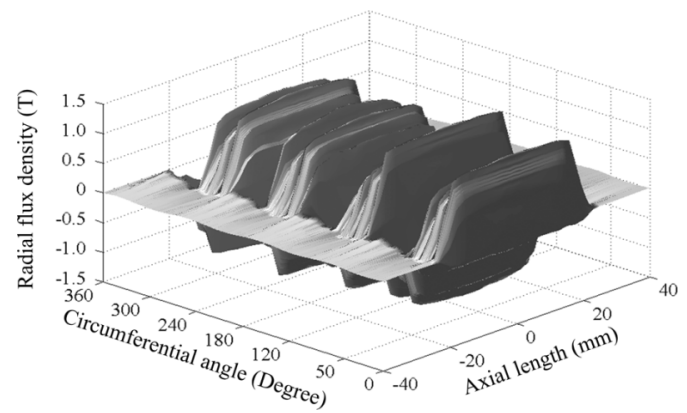

Fig. 2. 3D radial flux density in inner airgap of CMGRM.

exhibits outward-concentrated magnetic field, while the outer rotor exhibits inward-concentrated field. In order to provide a fair comparison, the two CMGs are with the same outside diameter of $214 \mathrm{~mm}$ and axial length of $40 \mathrm{~mm}$.

In order to investigate the end-effect of the CMGs, the 3D-FEM is engaged to calculate the radial flux density distributions of both CMGs. Fig. 2 shows the 3D radial flux density in the inner airgap of the CMGRM, in which the active part of the gear is from $-20 \mathrm{~mm}$ to $20 \mathrm{~mm}$ along the axial length. It can be seen that there exists severe leakage flux in the adjacent air space of the two ends. The same phenomenon can be observed in the CMGHM. This end-effect adversely affects the torque transmission ability of the CMGs.

By holding the inner rotor still and incrementally rotating the outer rotor, the torque-angle curves obtained from the 2D-FEM and 3D-FEM are shown in Fig. 3. It can be seen that the pull-out torques of the CMGHM obtained from the 2D-FEM are $51.7 \mathrm{Nm}$ (inner rotor) and $221.1 \mathrm{Nm}$ (outer rotor), while those obtained from the 3D-FEM are $37.3 \mathrm{Nm}$ (inner rotor) and 159.2 Nm (outer rotor). Moreover, the pull-out torques of the 


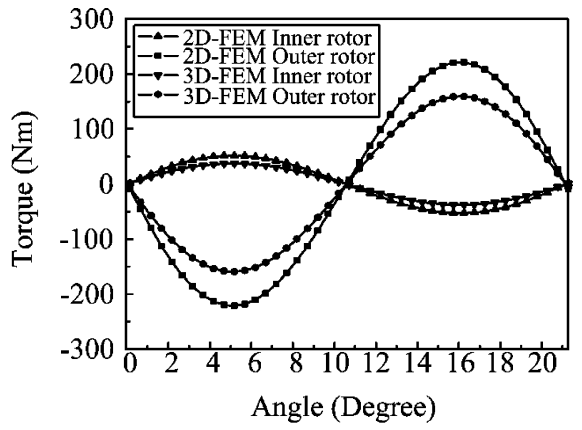

(a)

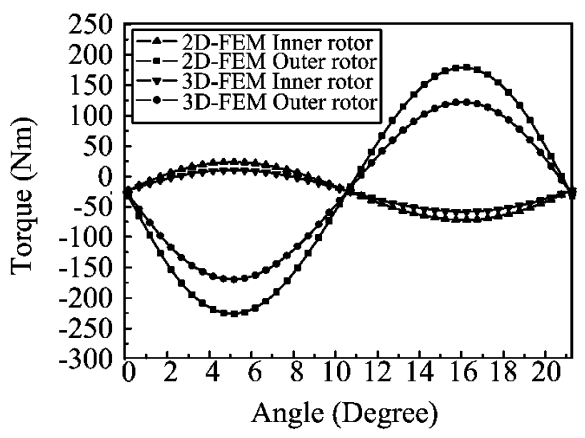

(b)

Fig. 3. Torque-angle curves. (a) CMGHM. (b) CMGRM.

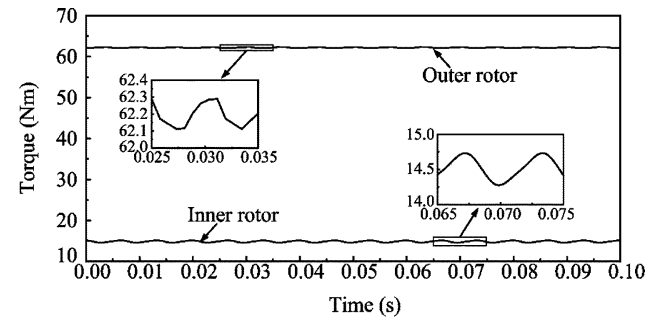

(a)

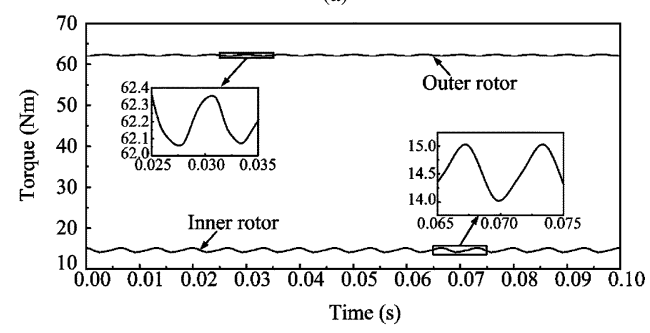

(b)

Fig. 4. Steady-state torque transmission. (a) CMGHM. (b) CMGRM.

CMGRM obtained from the 2D-FEM are $45.5 \mathrm{Nm}$ (inner rotor) and 195.2 $\mathrm{Nm}$ (outer rotor), while those obtained from the 3D-FEM are $33.1 \mathrm{Nm}$ (inner rotor) and $139.7 \mathrm{Nm}$ (outer rotor). Thus, the CMGHM can offer $14 \%$ higher pull-out torques than the CMGRM. By keeping the rotational speeds of the inner and outer rotors equal to $400 \mathrm{rpm}$ and $-1700 \mathrm{rpm}$, respectively, the steady-state torque transmission waveforms are depicted in Fig. 4 in which both CMGs are with the same load torque. It can be seen that the CMGHM can offer lower torque ripples than the CMGRM.

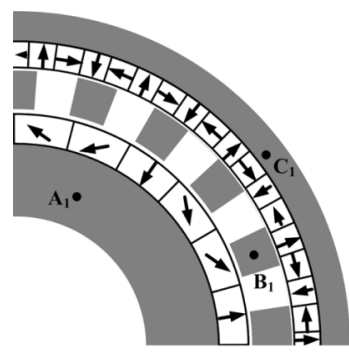

(a)

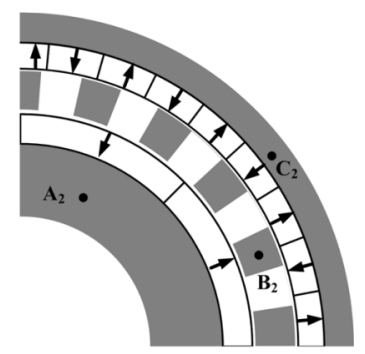

(b)
Fig. 5. Observation points. (a) CMGHM. (b) CMGRM.

\section{IRON LOSSES}

The iron losses occurred in the iron yokes of the rotors and the stationary ring are an important performance criterion of the CMGs. Since there are plentiful rotating magnetic field harmonics during the operation of the CMGs, those empirical approaches are no longer acceptable to estimate the iron losses. So, the use of FEM to evaluate the iron losses of the two CMGs is adopted [9]. After obtaining the magnetic field distribution, the flux density waveform of each finite element is decomposed into various harmonics by using the Fourier series. Then, the total iron losses, namely the summation of the eddy current loss $P_{e}$ and the hysteresis loss $P_{h}$, can be expressed as:

$$
\begin{aligned}
& P_{e}=\oint \sum_{n} \rho K_{e}(n f)^{2}\left(B_{n r}^{2}+B_{n t}^{2}\right) d V \\
& P_{h}=\oint \sum_{n} \rho K_{h}(n f)\left(B_{n r}^{2}+B_{n t}^{2}\right) d V
\end{aligned}
$$

where $\rho$ is the density of the iron core, $n$ denotes the order of the harmonics, $f$ is the frequency of the fundamental harmonic, $V$ is the volume of the iron core, $B_{n r}$ and $B_{n t}$ are respectively the radial and tangential components of the $n$th harmonic, and $K_{e}$ and $K_{h}$ are the coefficients of iron losses.

As shown in Fig. 5, some observation points are set in which the subscripts 1 and 2 refer to the CMGHM and CMGRM, respectively. Namely, the points $A_{1}$ and $A_{2}$ are on the iron yoke of the inner rotor, $B_{1}$ and $B_{2}$ are on the ferromagnetic segment of the stationary ring, and $\mathrm{C}_{1}$ and $\mathrm{C}_{2}$ are on the iron yoke of the outer rotor. Figs. 6, 7, and 8 show the flux density waveforms and loci of the observation points $A_{1}$ and $A_{2}, B_{1}$, and $\mathrm{B}_{2}$ as well as $\mathrm{C}_{1}$ and $\mathrm{C}_{2}$, respectively. It can be seen that because of the self-shielding magnetization of Halbach arrays, the CMGHM exhibits lower flux densities in the iron yokes of the rotors than the CMGRM. Moreover, although the CMGHM exhibits stronger field intensity in the ferromagnetic segment, the higher order harmonics can be suppressed due to the feature of near-sinusoidal distribution of the Halbach arrays. These can benefit the reduction of the iron losses. Finally, the iron losses at different rotational speeds are obtained as shown in Fig. 9. It can be seen that when the outer rotor rotates at $200 \mathrm{rpm}$, the iron losses in the CMGHM and the CMGRM are $40.4 \mathrm{~W}$ and $54.2 \mathrm{~W}$, respectively. Thus, the CMGHM can offer about $25 \%$ lower iron losses than the CMGRM.

\section{EXPERIMENTAL VERIFICATION}

As shown in Fig. 10, the two prototypes are built for experimental verification. Fig. 11 shows the measured torque-angle 


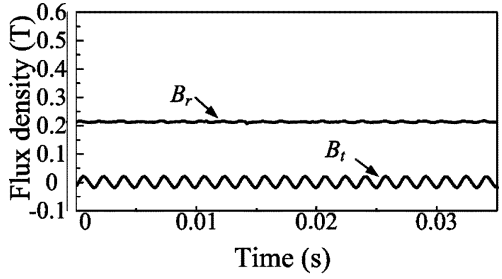

(a)

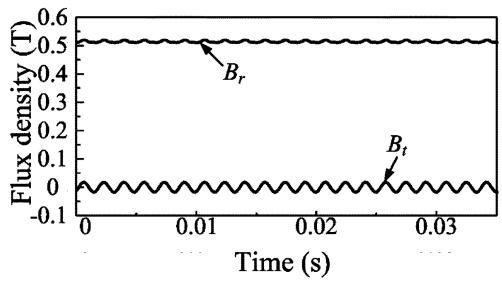

(b)

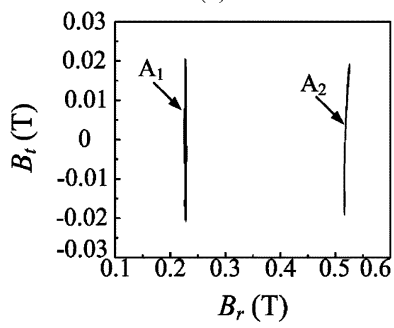

(c)

Fig. 6. Radial and tangential flux densities at observation points $A_{1}$ and $A_{2}$. (a) Waveforms at $A_{1}$. (b) Waveforms at $A_{2}$. (c) Loci at $A_{1}$ and $A_{2}$.

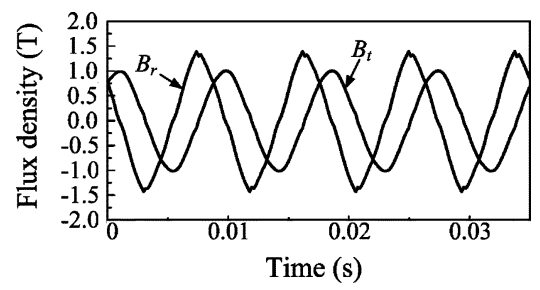

(a)

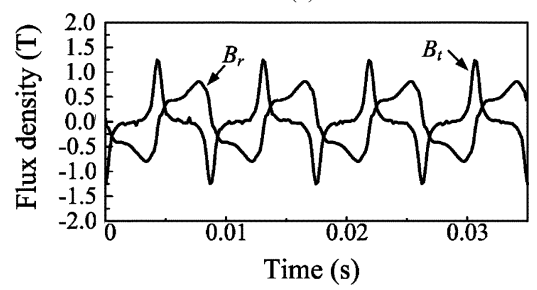

(b)

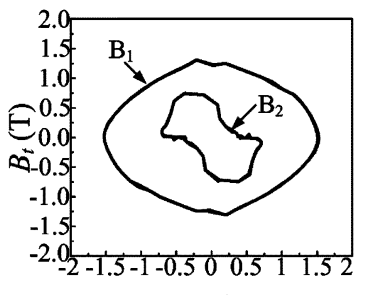

$B_{r}(\mathrm{~T})$

(c)

Fig. 7. Radial and tangential flux densities at observation points $\mathrm{B}_{1}$ and $\mathrm{B}_{2}$. (a) Waveforms at $B_{1}$. (b) Waveforms at $B_{2}$. (c) Loci at $B_{1}$ and $B_{2}$.

curves of both CMGs. It can be found that the measured pull-out

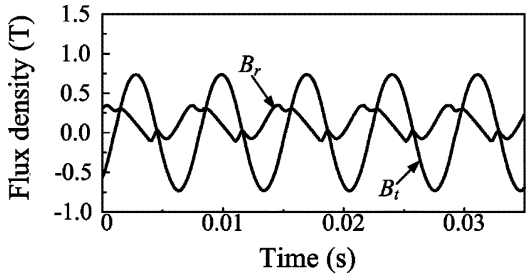

(a)

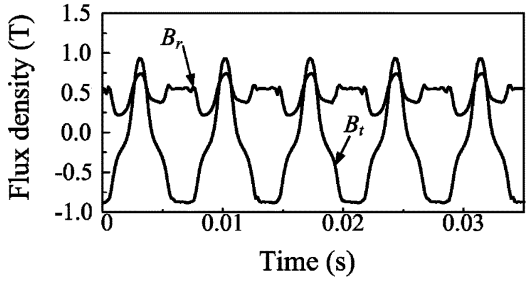

(b)

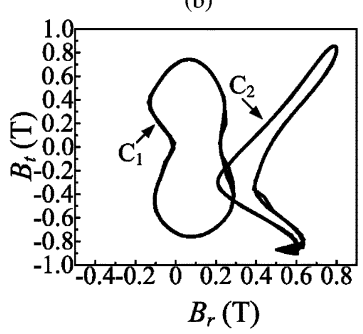

(c)

Fig. 8. Radial and tangential flux densities at observation points $\mathrm{C}_{1}$ and $\mathrm{C}_{2}$. (a) Waveforms at $\mathrm{C}_{1}$. (b) Waveforms at $\mathrm{C}_{2}$. (c) Loci at $\mathrm{C}_{1}$ and $\mathrm{C}_{2}$.

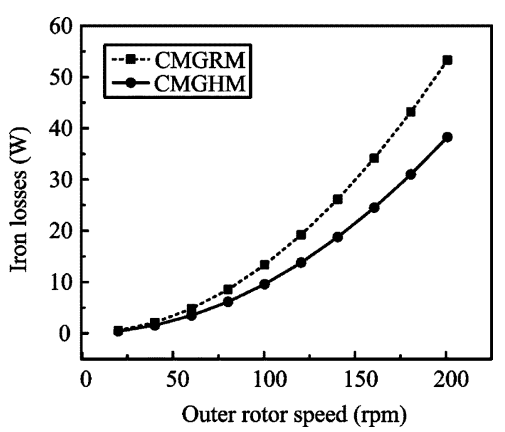

Fig. 9. Comparison of iron losses at different speeds.

torques of the CMGHM are $36.9 \mathrm{Nm}$ (inner rotor) and $155.8 \mathrm{Nm}$ (outer rotor), while that of the CMGRM are $32.4 \mathrm{Nm}$ (inner rotor) and $137.2 \mathrm{Nm}$ (outer rotor). They closely agree with the analysis results obtained from the 3D-FEM as shown in Fig. 3. In fact, both of these 3D-FEM and measured results match with the theoretical gear ratio of 17:4.

Moreover, Fig. 12(a) shows the measured no-load losses at different speeds, which verifies that the CMGHM has lower no-load losses than the CMGRM. It is interesting to note that when the outer rotor rotates at $200 \mathrm{rpm}$, the no-load losses in the CMGHM and CMGRM are $173 \mathrm{~W}$ and $196 \mathrm{~W}$, respectively. Compared with the results of $40.4 \mathrm{~W}$ and $54.2 \mathrm{~W}$ as shown in Fig. 9, it can be deduced that the corresponding mechanical losses are $132.6 \mathrm{~W}$ and $141.8 \mathrm{~W}$ which are quite similar and significant. Finally, Fig. 12(b) plots the measured transmission efficiency of both CMGs at different load torques and different 


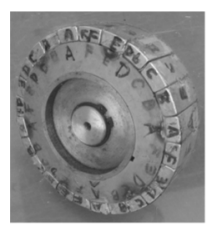

(a)

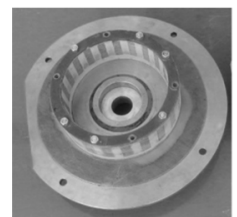

(d)

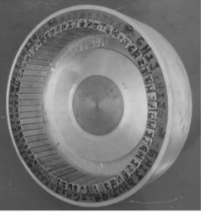

(b)

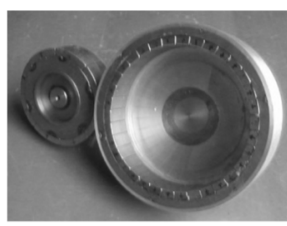

(c)

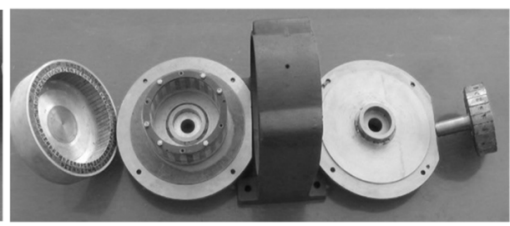

(e)
Fig. 10. Prototypes. (a) Inner rotor of CMGHM. (b) Outer rotor of CMGHM. (c) Inner and outer rotors of CMGRM. (d) Stationary ring. (e) Assembly.

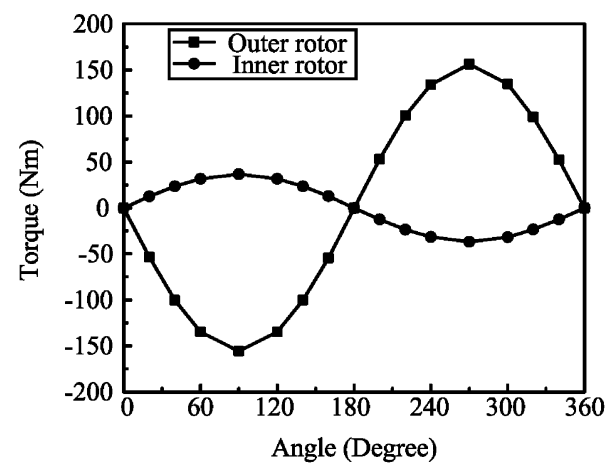

(a)

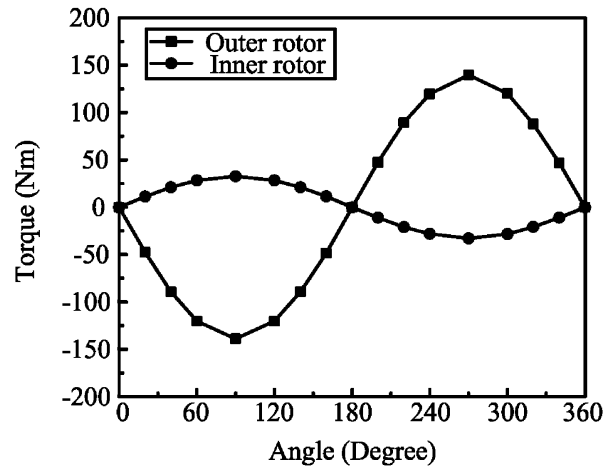

(b)

Fig. 11. Measured torque-angle curves. (a) CMGHM. (b) CMGRM.

rotational speeds. It further confirms that the CMGHM always offers higher efficiency than the CMGRM.

\section{CONCLUSION}

Two latest CMGs, namely the CMGHM and CMGRM, have been quantitatively compared. By using the 3D-FEM, the endeffect and the performances of these CMGs are investigated. The analysis results confirm that the CMGHM can offer higher pull-out torque, lower torque ripple and lower iron losses than the CMGRM. After prototyping both CMGs, the experimental results are given to verify the analysis results.

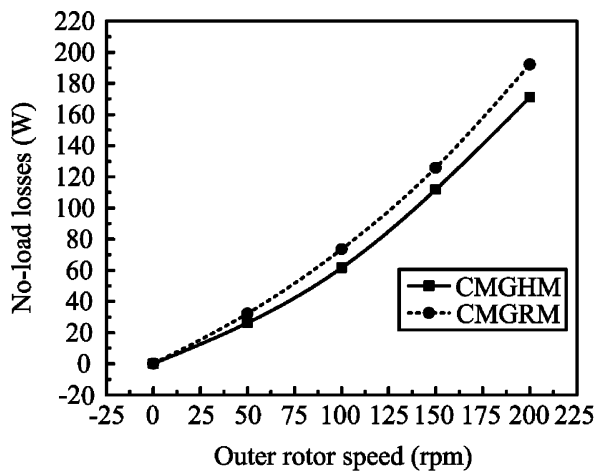

(a)

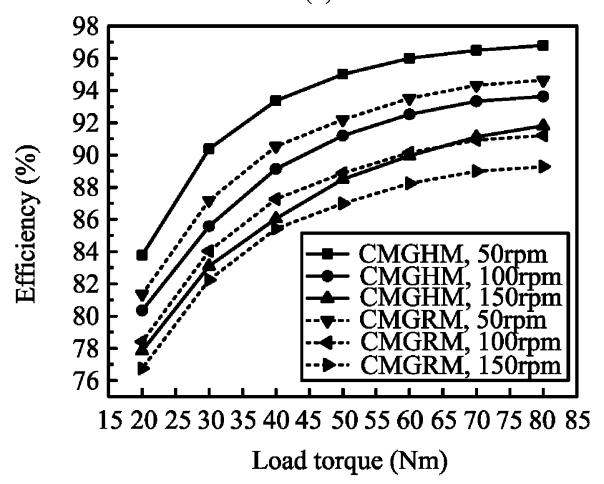

(b)

Fig. 12. Measured loss characteristics. (a) No-load losses. (b) Efficiency.

\section{ACKNOWLEDGMENT}

This work was supported by the Research Grants Council, Hong Kong Special Administrative Region, China, under Grant HKU 7105/07E.

\section{REFERENCES}

[1] K. Atallah and D. Howe, "A novel high-performance magnetic gear," IEEE Trans. Magn., vol. 37, no. 4, pp. 2844-2846, Jul. 2001.

[2] K. T. Chau, D. Zhang, J. Z. Jiang, and L. Jian, "Transient analysis of coaxial magnetic gears using finite element comodeling," J. Appl. Phys., vol. 103, no. 7, pp. 07F101-, Jan. 2008.

[3] X. Liu, K. T. Chau, J. Z. Jiang, and C. Yu, "Design and analysis of interior-magnet outer-rotor concentric magnetic gears," J. Appl. Phys. vol. 105, no. 7, pp. 07F101-, Feb. 2009.

[4] L. Jian and K. T. Chau, "Analytical calculation of magnetic field distribution in coaxial magnetic gears," Prog. Electromagn. Res., vol. 92, pp. 1-16, 2009.

[5] K. T. Chau, D. Zhang, J. Z. Jiang, C. Liu, and Y. J. Zhang, "Design of a magnetic-geared outer-rotor permanent-magnet brushless motor for electric vehicles," IEEE Trans. Mag., vol. 43, no. 6, pp. 2504-2506, Jun. 2007.

[6] L. Jian, K. T. Chau, and J. Z. Jiang, "A magnetic-geared outer-rotor permanent-magnet brushless machine for wind power generation," IEEE Trans. Ind. Appl., vol. 45, no. 3, pp. 945-962, May/Jun. 2009.

[7] J. Choi and J. Yoo, "Design of a Halbach magnet array based on optimization techniques," IEEE Trans. Magn., vol. 44, no. 10, pp. 2361-2366, Oct. 2008.

[8] L. Jian and K. T. Chau, "A coaxial magnetic gear with Halbach permanent magnet arrays," IEEE Trans. Energy Conv., to be published.

[9] H. Domeki, Y. Ishihara, C. Kaido, Y. Kawase, S. Kitamura, T. Shimomura, N. Takshashi, T. Yamada, and K. Yamazaki, "Investigation of benchmark model for estimating iron loss in rotating machine," IEEE Trans. Magn., vol. 40, no. 2, pp. 794-797, Mar. 2004. 